\title{
Correction to: Literature Reviews
}

\author{
Ana Paula Cardoso Ermel $[$, D. P. Lacerda $₫$, \\ Maria Isabel W. M. Morandi $\odot$, and Leandro Gauss
}

\section{Correction to:}

\section{A. P. Cardoso Ermel et al., Literature Reviews, https://doi.org/10.1007/978-3-030-75722-9}

In the original version of the book, the following belated corrections have been incorporated:

In chapter "Literature Analysis", missing bullets have been updated in Figure 4.12. In chapter "Literature Synthesis", Figure 5.1 has been moved to section 5.2.9 Grounded Theory from 5.2.10 Meta-Ethnography.

In chapter "Literature Grounded Theory (LGT)", figures are centred in Table 6.28 and the flow arrows are corrected in Figures 6.2, 6.5.

In chapter "Computational Tools for Literature Review, Analysis, and Synthesis", white space has been removed in Figure 7.1.

The erratum chapters have been updated with the changes.

The updated versions of the chapters can be found at https://doi.org/10.1007/978-3-030-75722-9_4 https://doi.org/10.1007/978-3-030-75722-9_5 https://doi.org/10.1007/978-3-030-75722-9_6 https://doi.org/10.1007/978-3-030-75722-9_7 